\title{
Reservoir Dam Safety Surveillance Mode in Switzerland and Its Experience Reference to China
}

\author{
Shichen ZHANG ${ }^{a, b}$ \& Zhaosheng WANG ${ }^{a, b} \& \mathrm{Li} \mathrm{Ji}^{\mathrm{c}}$
}

\author{
a, Dam Safety Management Center of the Ministry of Water Resources, Nanjing, Jiangsu, China; \\ b, Nanjing Hydraulic Research Institute, Nanjin, Jiangsu, China \\ c, Hydraulic Engineering College of Changsha University of Science \& Technology, Changsha, \\ China
}

\begin{abstract}
KEYWORD: Switzerland; dam safety; safety surveillance; reference
ABSTRACT: Dam safety surveillance model in Switzerland is a classic example of good combination of traditional way and modern concept. There is no dam failure in history of Switzerland. Swiss dam safety surveillance mode is highly praised by International Commission on Large Dams and is of enlightening and reference significance to the safety management of reservoir dams in China. Based on introducing the basic information of dams in Switzerland and safety surveillance concept, this paper systematically analyzes the safety surveillance mode of dams in Switzerland, digs the safety surveillance characteristics of dams in Switzerland. Through contrastive analysis of the dam safety management modes in China and Switzerland, building safety culture, defining management responsibilities, enhancing surveillance and inspection, making innovative management mode, attaching importance to emptying requirements, perfecting metal structure testing system and a series of experience in Switzerland are put forward for China's reference.
\end{abstract}

\section{BASIC INFORMATION OF RESERVOIR DAMS IN SWITZERLAND}

There are 1200 reservoirs of all kinds in Switzerland. Most of them are dominated by the types of retaining water and generating electricity and those in minority are oriented by flood protection, irrigation, sand interception and other social benefits. Switzerland began building industrial dams in the beginning of 19th century, damming industry had further development in 1930s, and reached its climax in 1950 1970 with a batch of high dams and large reservoirs built in succession ${ }^{[1]}$. Grande Dixence built in 1961, with dam height of 285m, is still keeping the highest world record of concrete gravity dam at present.

Reservoirs in Switzerland can be classified into small reservoirs and large ones according to the project scale ${ }^{[2]}$. Large reservoirs refer to the reservoirs with dam height above $25 \mathrm{~m}$ or dam height above $15 \mathrm{~m}$ and storage capacity above $50,000 \mathrm{~m}^{3}$, or dam height above $10 \mathrm{~m}$ and storage capacity above $100,000 \mathrm{~m}^{3}$, or storage capacity above $500,000 \mathrm{~m}^{3}$; small reservoirs refer to those with dam height above $10 \mathrm{~m}$ or dam height above $5 \mathrm{~m}$ and storage capacity no less than $50,000 \mathrm{~m}^{3}$. There are 227 large reservoirs in Switzerland, and the rest 1000 ones are small reservoirs. In order to further enhance the safety surveillance of dams in lager scales, the concept of outsize reservoirs is further emphasized within the large reservoir scope in management. This kind of reservoirs refers to the reservoirs with dam height no less than $40 \mathrm{~m}$ or dam height no less than $10 \mathrm{~m}$ and storage capacity no less than $1,000,000 \mathrm{~m}^{3}$.

Classified according to dam type, gravity dams account for 35\%, earth dams account for $32 \%$, arch dams account for $24 \%$, buttress dams account for $1 \%$ and the rest $8 \%$ are water gates, which are also supervised as reservoir dams.

Switzerland preliminarily established the legal framework for dam safety management in 1943. Dam Law in 1998 (WRFA) ${ }^{[3]}$ was enforced and was perfected year by year hereafter and reaches current situation. At present, the main laws and regulations on safety surveillance of reservoir dam in Switzerland are Dam Law and Dam Regulation (WRFO) ${ }^{[4]}$. To carry out Dam Regulation, Federal Office of Energy published dam safety criterion, structure safety, flood control safety, earth- 
quake-resistance standard assessment, dam monitoring and maintenance, contingency plan and a series of guidelines.

Grading supervision similar to that of China is also implemented for reservoir dam safety in Switzerland. 227 large reservoirs are in the charge of Swiss Federal Office of Energy (SFOE) and the rest 1000 small reservoirs are in the charge of state government reservoir dam safety supervision organs. Swiss Federal Office of Energy (SFOE) exercises indirect supervision on the supervisory behaviors of small reservoirs conducted by governmental dam safety supervision organs..

\section{SAFETY CONCEPT OF RESERVOIR DAM IN SWITZERLAND}

The safety management objective of dams in Switzerland is to control initial, operation and residual risks and ensure the dam safety and effective respond to emergency circumstances ${ }^{[5]}$. This concept can be realized in three aspects (see Fig.1).

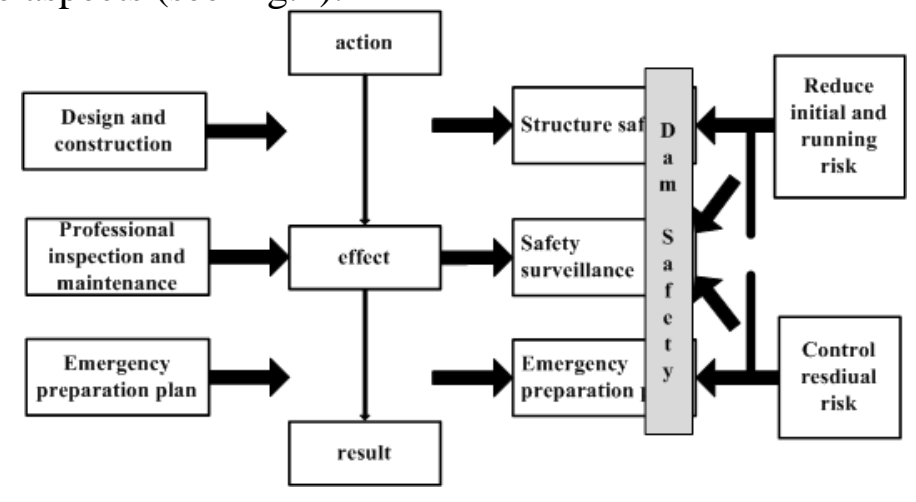

Fig.1 Safety Concept of Reservoir Dam in Switzerland

(1) Structure safety. Supervision organs examine construction plans in pre-construction phase of dams, make whole process supervision of dam construction behaviors in the construction and implementation, and examine initial filling to ensure the engineering structure safety and provide foundation for the official operation in later phase.

(2) Safety surveillance. In the whole-life process of dams, the owners must exercise effective safety surveillance, find defects and abnormal forms in time, prevent possible dangers, and submit safety report to the supervision organs on a regular basis. The supervision organs should have dam monitoring under control and comply with the relevant legal provisions, should meet dam safety requirements at any time, and may require the owners to take appropriate measures to ensure the dam structure safety.

(3) Emergency plan. Although safety surveillance is carried out continuously, the dam safety cannot be ensured forever due to inevitable abnormal behaviors, natural accidents or destructive activities, etc. Once unexpected accidents happen, all measures must be taken to avoid endangering the safety of personnel, property and environment. Therefore, the contingency plans should be prepared to prevent the safety problems that may appear to control the residual risks.

\section{SURVEILLIANCE MODE OF SWITZERLAND}

\section{Dam safety surveillance system}

Swiss dam safety surveillance system is divided into four layers (see Fig.2), respectively are L1 dam caretakers, L2 qualified engineers, L3 senior experts and L4 supervisors. Through the continuous inspection and tests of L1 dam caretakers, monitoring and assessment of L2 qualified engineers each year or at any time, deep safety assessment of L3 senior experts once every five years, supervision and management of L4 supervisors and interaction between the personnel of all layers and their constant operation, this system guarantees the continuous effect of dam safety surveillance. 


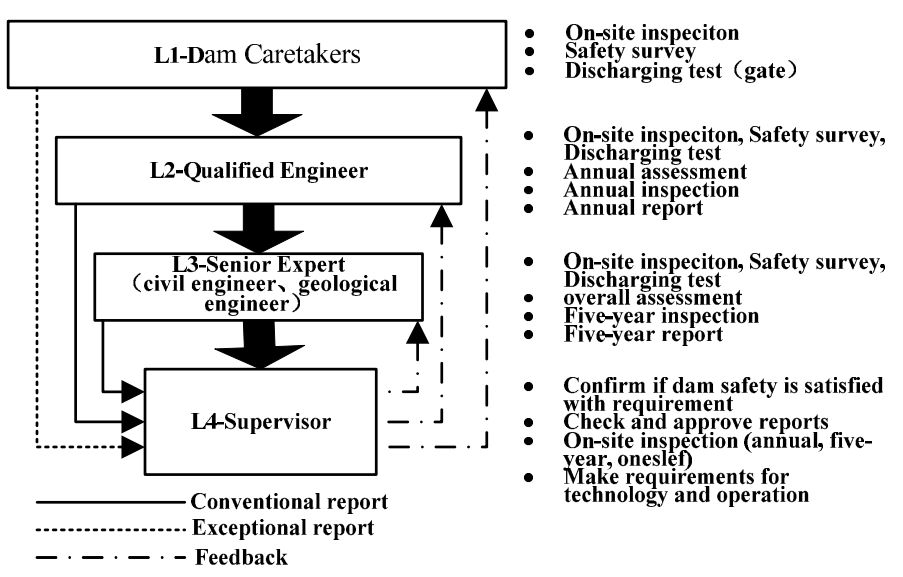

Fig.2 Swiss Dam Safety Surveillance System

L1 personnel are dam caretakers. They need to know what they should do and be familiar with management equipment performance, provide timely and accurate safety monitoring, site inspection, water drawing off test information for the personnel of other layers.

L2 personnel are qualified engineers. They play a key role, and carry out site inspection of legal frequency within each year, take part in water drawing off test, are responsible for the surveillance and training of L1 personnel and feeding the dam safety status back to owners and relevant personnel, find problems in time, put forward solutions and submit annual reports of dam safety to the owners and L4 supervisors in the second year regularly.

L3 personnel are senior experts. The personnel of this kind are few. They carry out site inspection of legal frequency, are responsible for surveillance and training of L2 qualified engineers and L1 dam caretakers, comprehensively analyze engineering safety conditions, put forward necessary maintenance, reinforcement and monitoring or further monographic studies and other measures and suggestions, and submit 5-year reports to the owners and L4 supervisors in the sixth year regularly. L4 personnel refer to the personnel in supervision organs. They are responsible for the supervision of safety surveillance behaviors and results of personnel at the layers from L1 to L3, carrying out dam site inspection of legal frequency, examining various safety reports, making requirements on dam safety technology and operation measures, and putting forward requirements on reducing reservoir water level or other preventive measures (if necessary).

The aforesaid surveillance system of four layers has a certain corresponding relation with the safety operation and management system of reservoir dam in China. Swiss L1 dam caretakers correspond with reservoir running and management operating personnel in China, who are responsible for the site inspection, site monitoring, operation and scheduling, etc. in the daily management of dam safety operation; Swiss L2 qualified engineers correspond with the department heads undertaking dam safety management tasks in China, such as the technology chief engineer of some reservoir or the section chief of engineering supervision section, etc. Swiss L4 supervisors correspond with dam safety supervisors in China. Because the safety control system of reservoir dam in China is relatively complex and implementing supervision by competent water administrative department at all levels jointly with departments concerned is adopted as the main form, the supervisors in different industries have different expression forms. Water conservation system supervision organs refer to the Ministry of Water Resources and departments (bureaus) of water conservancy (affairs) at all levels, and energy industry refers to National Energy Administration, all resident agencies and Dam Safety Supervision Center, National Entergy Administration.

Swiss four-layer dam safety regulation system enjoys clear division of responsibilities, clear duties, is easy to comprehend and implement and is conducive to efficient implementation of work. L2 qualified engineers in the system are the connecting link between the preceding and the following and are the key figures in mastering the dam comprehensive information and knowing dam safety status. 


\section{Contents of dam safety surveillance}

The contents of safety surveillance of dams in Switzerland mainly include the three aspects of safety monitoring, site inspection, and water drawing off tests so as to complete instrument measuring and reading, visual inspection, equipment testing and other work to ensure the engineering, metal structure and electromechanical devices in safe and reliable condition.

\section{Key surveillance system measures}

(1) Daily inspection

Daily inspection is completed by L1 dam caretakers, who carry out uninterrupted safety monitoring, site inspection and water drawing tests according to surveillance system. Switzerland does not think that the caretakers need high qualification while they should have responsibility, patience, communication ability and other basic qualities. There are many L1 caretakers unable to use computer, however, they still can complete dam safety operation and management tasks well. Stimulating the work motivation of L1 caretakers, enriching safety concept through case learning, enhancing educational training (especially valuing the training method of cultivating the new by the old similar to that in China), paying attention to knowledge update, letting them understanding why this post is set, and the data information obtained in daily management is of great significance to the analysis and study of dam safety form of the personnel from L2 to L4.

(2) Annual report

L2 qualified engineers are responsible for preparing dam safety annual report and should submit to the dam supervision organs within the first 6 months in the second year. The dam safety annual report mainly include gate operational testing, safety monitoring, site inspection and other circumstances, explaining gate testing, safety monitoring, site inspection and other operation situations, analyzing the surveillance results of dams, ancillary facilities, bank slope of reservoirs, etc., assessing whether the projects are safe, putting forward necessary maintenance, reinforcement and monitoring and other measures and suggestions. The characteristic of Swiss dam safety annual reports is clear at a glance, simple and concise, usually with only 20 30 pages. The simplest report has only 1 page for a small dam.

One of the important foundations of annual report is annual inspection, which is completed under the leadership of L2 engineers. Annul inspection reports are prepared for some reservoirs according to the annual inspection situation as the supporting materials of dam safety annual reports. For annual site inspection reports, all information related to dams must be included with the spatial scale including dam, water discharge and delivery structure, operating equipment and area around and with contents including characteristic description of area inspected, description of dam seepage or deformation, clear development tendency and new problems, necessary graphics, photos, equipment maintenance, renovation and transformation, etc., such as replacement of a pressure gage, renovation and transformation of power line, which should be fully written into the annual site inspection reports.

It is very important that, not only should L2 qualified engineers propose an annual report at the end of the year and in the beginning of the second year, but also they need to make analysis on the observational data each month (see Fig.3), including any major abnormity, whether any abnormal variation trend appears, whether there are some rapidly increasing measuring values and participation in earthquake ${ }^{[6]}$, flood and other special inspections. If encountering abnormal safety status, they should quickly inform all personnel concerned of paying attention to the issue. If encountering any extremely abnormal monitoring result when they cannot make decision, L2 qualified engineers should report to L3 senior experts and L4 supervisors. In addition, monitoring regulations also need to be formulated. If observation instrument is damaged and maintenance or replacement is needed, they can complete the regular and simple work by themselves and complete update and maintenance work along with L1 caretakers (such as measuring weir installation). As for the special instrument, they need to report the relevant information to L3 senior experts and L4 supervisors before design and installation. Skillfully mastering instrument is very important to L2 qualified engineers and helping L1 caretakers to be familiar with instrument characteristic and monitoring method is not only a paper work for completing a report. 


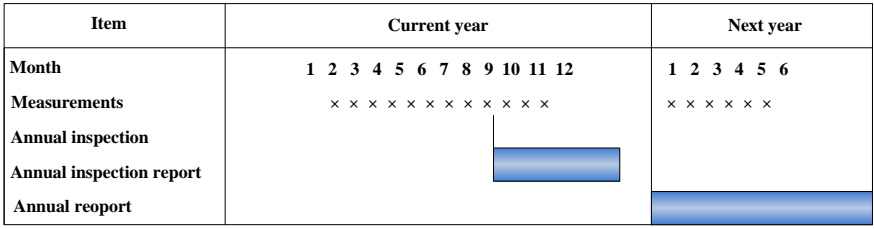

(1) Surveillance Course of L2 Qualified Engineer

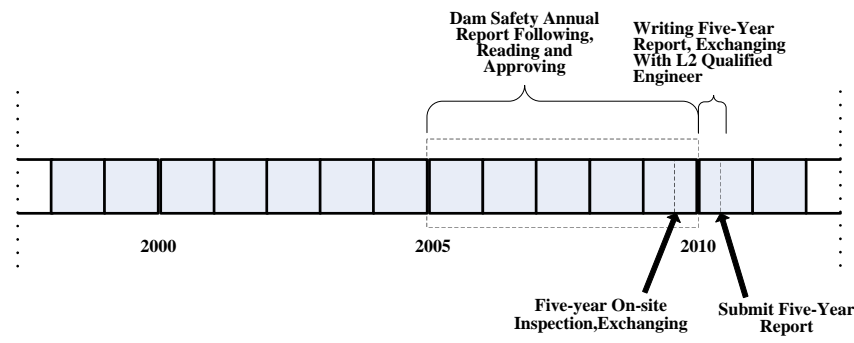

(2) Five Year Safety Surveillance Courses of L3 Senior Expert From 2005 to 2009

Fig.3 Diagram of Dam Safety Surveillance Course of L2

Qualified Engineer and L3 Senior Expert

(3) 5-year report

In accordance with the Dam Law and Dam Regulation, for outsize reservoirs, L3 senior experts should carry out one site inspection every five years, conduct dam safety assessment and put forward 5-year assessment reports on dam safety, and the 5-year reports must be submitted within the first 9 months in the sixth year (see Fig.3). This work is similar to the regular dam safety assessment of reservoirs and hydropower stations in China.

The 5-year report is similar to annual report, clearing whether the dam project status is normal and the suggestions for the next 5-year supervision period mainly based on the evaluation and analysis on safety monitoring, site inspection, and water drawing off tests, for example, whether it is necessary to make adjustment on the observation procedure in the aspect of safety monitoring, whether it is necessary to make renovation and transformation on monitoring facilities, or whether it is necessary to additionally set special monitoring facilities (such as reservoir area glacier displacement monitoring) and so forth, and formulating monitoring schedule for the subsequent 5 years. The 5year reports made by L3 experts are implemented based on the annual reports. L3 experts should judge the results and records of L1 caretakers and L2 engineers with critical eyes.

L3 experts are the long-term technology consulting experts and are the technology consulting experts of whole 4-layer surveillance system, functioning as a bridge connecting L2 and L4. If encountering problems too difficult to solve, personnel of L1, L2 and L4 seek for solutions to L3 experts. If L3 experts cannot solve them either, external specialists will be specially invited for help, and monographic studies will be carries when necessary, such as, reservoir area glacial sliding subject analysis, hydrological analysis, anti-earthquake analysis, etc. Swiss L3 experts are responsible for corresponding dam safety consultation for long term. Although there is report quality lifelong system in our county, however, consultation work is only carried out during the implementation period of dam safety evaluation project while technology consultation is seldom carried out in general after the completion of project.

(4) Supervision and inspection

Provisions on supervision and inspection of L4 supervisors are clear in Switzerland. In accordance with the Dam Law and Dam Regulation, in addition to the participation in 5-year site inspection of L3 senior experts, L4 supervisors require independent site supervision and inspection on outsize reservoirs at least once every five years, large reservoirs once every three years and small reservoirs once every five years, (the provisions on the inspection frequency here are determined through comprehensively considering the frequency of inspection needing to be carried out by personnel of L1, L2 and L3). In the meantime, all kinds of safety reports are examined and verified, requirements on dam safety technology and operation measures are made, and requirements on reducing 
reservoir water level or other preventive measures (if necessary) are put forward. If L4 supervisors don't think that L2 engineers or L3 experts reported by the owners are competent for corresponding work, they have one-vote veto power and are entitled to require replacement.

\section{Dam safety surveillance characteristic}

(1) Dam safety surveillance in Switzerland is clear in concept, perfect in system, clear in responsibilities and smooth in mechanism with regulation reaching the degree of virtuous cycle. The safety supervision and management of dams in Switzerland are completely independent; perfect regulatory system is set up for the whole cycle from planning and design, construction and implementation, pilot running, official running, reinforcement and reconstruction, decommissioning and demolition; the responsibilities supervision and management personnel as well the governments at all levels are very clear and are specified by law; the mechanism is smooth, reflecting the work trace of the supervisors.

(2) Paying attention to dam safety monitoring. The concept of safety monitoring of dams in Switzerland is "simple, reliable, durable in use, not blindly worshiping automatic monitoring"[7]; objectives of monitoring design are definite, large quantity is not needed for monitoring point with simple but reasonable layout and meanwhile it is emphasized that monitoring parts, monitoring projects and measure point quantity should be chosen according to the project characteristic or individuality. Monitoring facilities operate completely and are maintained in place, the facility maintenance is good and importance is attached to manual-comparison measurement; monitoring information can be analyzed in time in annual reports and 5-year reports and the analysis results are used for guiding dam safety operation and management. Swiss Matmark Reservoir is a large reservoir with the total storage capacity of $100,000,000 \mathrm{~m}^{3}$. Its observation facility of seepage pressure of earth and rockfill dam was inbuilt in 1960s, the device periodically cleaning sediment in piezometric tube was set for the convenience of later maintenance and is still intact up to now. Switzerland is powerful nation of producing precise instrument, has Leica Instrument and other advanced monitoring instrument production companies. However, automatic monitoring is not excessively pursued for Swiss dam safety monitoring. Automatic acquisition system is only set for the dam body, temperature, seepage pressure, seepage discharge, dam foundation multipoint shift and other observation items with the dam safety form can be independently judged to improve the efficiency of monitoring.

(3) Importance is attached to site inspection and site monitoring and the credibility of monitoring data is emphasized. Great importance is attached to monitoring data reliability and automatic monitoring data for manual calibration for Swiss dam safety monitoring. Dam Regulations stipulates, manual-comparison measurement should be carried out at least once every month for large reservoirs, and manual-comparison measurement should be carried out at least once a year for other reservoirs. In recent years, the reservoir dam safety monitoring work had remarkable progress in China. However there are several notable phenomena: safety monitoring automatic system is blindly relied on and manual-comparison measurement is overlooked; monitoring design guiding ideology is less scientific with quality not high; the operation and maintenance of monitoring facilities are not in place, the serviceability rate of instrument lasting more than 10 years is not high; the reliability of monitoring data is insufficient and analyses are not in place.

(4) Importance is attached to the completeness and continuity of observational data. Importance is greatly attached to the accumulation and analysis of dam safety monitoring data and attention is paid to maintaining the continuity and consistency of the contents of dam safety monitoring in Switzerland. In the dam annual safety reports and deep dam annual safety assessment reports once every five years, monitoring data is dominated by analysis and assessment of dam form. For example, the conclusion of the existence of irreversible deformation in dam foundation on the left side of Toules arch dam (the biggest dam height of $86 \mathrm{~m}$ ) was derived from the analysis and judgment made based on the observational data for as long as 38 years.

(5) Importance is attached to the dynamic management of surveillance personnel qualification. The qualification of L2 qualified engineers and L3 senior experts is implemented to persons instead of an entity; the dynamic management of surveillance personnel qualification is strict and the experts competent for surveillance work are not easily replaced, that is, fixed principle is adopted for the 
experts employed at all levels. Unless the experts are found not competent for surveillance work, the supervisors at L4 is entitled to require replacement.

(6) Importance is attached to water drawing off test. Water drawing off test, site inspection and safety monitoring are jointly taken as the main contents of dam safety surveillance and the must-do tasks in the corresponding circumstances and no-need tasks in the corresponding circumstances are defined, and how-to-do (such as test working conditions, test methods, test records and test report, etc.) and other contents are explicitly stipulated in Dam Regulations at the statute level to ensure the reliable running of gate control system.

(7) Importance is attached to reservoir emptying capability. It is clearly stipulated in Dam Law that each reservoir in Switzerland should have emptying capability. It is required that water drawing off test must be carried out for emptying reservoir and required ensuring conditions are conditionally provided in emergency circumstances, maintenance and safety inspection..

\section{EXPERIENCE REFERENCE FOR SAFETY SURVEILLANCE OF DAMS IN SWITZERLAND}

Four-layer safety surveillance system established in Switzerland is clear in structure and is simple and practical, advocating dam safety management cultural construction and delicacy management, which is a classic example combining traditional modes and modern concept. Comparing the dam safety management systems in China and Switzerland, their main objective and framework is basically consistent. Swiss dam safety surveillance mode caters to the attention to the existing problems of dam safety management in the country paid by the Chinese experts and can provide a certain reference meanings for the dam safety management in China.

(1) Building reservoir dam safety culture

Experience has convinced Switzerland that ignorance, complacency and negligence are the important factors leading to dam failures, and it is very necessary to construct dam safety culture in organization and management. The dam safety culture is the generic term for safety concept and safety awareness about dams and all behaviors under the guidance, mainly including security ideas, behavior safety, system safety, technology security safety, etc. The safety culture of dams in Switzerland has been rooted in life and work, is rigorous and careful, strives for actual effect, and it vigor remains unshakable and becomes even firmer as time goes by. Dam surveillance mode is called as "four eyes" control culture (see Fig.4), that is, the surveillance mode with L1 automatic control, L2 (L3) hierarchical control, L4 external independent control.

owner
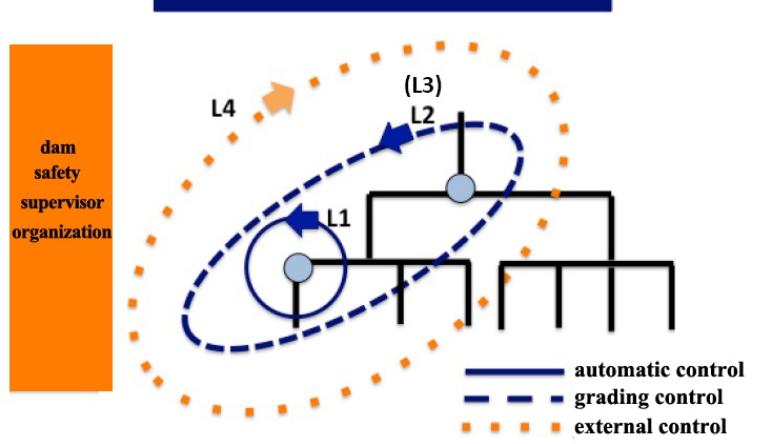

Fig.4 Dam Safety Surveillance Modes Existed in the Culture of Switzerland Dam Safety Management

(2) Perfecting dam safety surveillance contents

Switzerland emphasizes explicitly stipulating the responsibilities of all side, working procedure and information connection and other circumstances in dam safety surveillance. Reservoir management in China at present is mainly performing assets management and dispatching management at present, and importance shall be attached to safety surveillance and emergency management ${ }^{[8]}$.

(3) Enhancing dam safety monitoring inspection 
The surveillance personnel of L1 and L2 at basic level are responsible for reporting the abnormity of dams. However, the awareness of abnormity will be affected by human judgment ability. If judgment is only made by the personnel at basic level, it may be too late. It would be better if the information of change is reported continuously. In management, the permanent questions are: Can the dam safely operate in next year? Are there any abnormal signals? Will the dam be safe in the next five years? The wrong question is: Is this problem so serious enough to report (or inform boss)?

Aging phenomena of projects will appear after several years of operation in most cases. When safety loophole of what degree will appear and how the development and other circumstances are unpredictable. What is important is initiatively carrying out surveillance, performing stipulated procedures and task contents, including the annual measurement procedures, annual inspection reports, annual safety reports, deep safety assessment once every five years, and thematic safety assessment should also be carried when necessary. What counts is that measuring results cannot be blindly believed and clear verification procedures for measured data should be established ${ }^{[9]}$.

China can learn from the simple, reliable and durable safety monitoring concept in Switzerland, and study Swiss experience in the links of design, mode selection, testing, installation, maintenance, measurement and reading, analysis, applications, etc. Each step needs carefulness and the ultimate purpose is the analysis results of dam safety monitoring can be used for dam safety management. Site inspection system should be perfected, the qualification issue of site inspection experts of dam safety authentication needs to be defined, the importance of site inspection reports of dam safety authentication should be enhanced in safety authentication work and the reports should be submitted to supervision departments for examination or records.

(4) Innovation of dam safety surveillance mode

Safety regulation of dams in Switzerland is clear in concept and explicit in contents, and is worth for China to learn. China should define dam safety regulation responsibility division ${ }^{[10]}$, enhance the regulation awareness of supervision organs, perfect regulatory means, add dam safety annual report system, implement the regulation work into the whole lifecycle of dams, and generally propel and implement technology regulation. As for the reservoir dam safety operation and management, the relatively fixed system of consulting engineers for technicians similar to family doctor system should be explored and established, supervision organs should support, owner management entities establish good cooperation relations with relatively fixed L3 senior expert engineers so as to improve the technical ability of reservoir dam safety management, better give play to role of L3 experts and improve dam safety and management efficiency. Identification and examination of the qualification of personnel of L2 and L3 should be established, and the entity qualification requirement on the management of L3 senior expert engineers is transferred to the individual qualification requirement of each expert. For the small reservoirs lacking management conditions in general, learning from the managerial experience in Switzerland, several flexible management methods should be exercised, for example, Swiss combined type assets and operation management mode are very suitable reference for small reservoir safety management in China, including area centralized management, entrusted management, conducting management by substituting small units with large units.

(5) Perfecting L3 expert duty requirements

Dam safety evaluation method completed every 5 years or every 6 10 years by L3 experts should be perfected, comprehensive evaluation on observation facilities reliability and site inspection as well as safety monitoring result analysis work should be enhanced; necessary demand analysis should be carried out in advance before safety evaluation, thematic checking calculation and analysis work should be with discrimination, dam safety evaluation at present is changed from having large quantity and complexity into smaller quantity and better quality. It is defined in system that L3 experts must continuously bring the role of expert into play, make supervision, consultation and guidance of dam safety surveillance materials of L1 and L2 personnel well, perfect duty-powerbenefit distribution.

(6) Importance is attached to water drawing off test and reservoir emptying system 
The relevant system of reservoir dam safety management in China should clearly define water weepage and conveyance building equipment testing concept, put forward testing requirements combining the actual operation situations (working condition requirements, test contents, report requirements and inspection exemption conditions, etc.) of water weepage and conveyance building, perfect relevant system. Emptying requirements are not stipulated in laws, regulations and systematic criteria, and thus, reservoir water level cannot be quickly reduced under the catastrophic flood, great earthquake, terrorist attack and other emergency situations. The practice in Switzerland is instructive to China in this aspect.

\section{CONCLUSION}

Four-layer dam safety surveillance system established in Switzerland is clear in structure and is simple and practical, advocating dam safety management cultural construction and delicacy management. It is a classic example combining traditional modes and modern concept and can provide a certain reference meanings for the dam safety management in China.

\section{REFERENCES}

[1]SFOE. Swiss Committee on Dams [A]. Dams in Switzerland Source for Worldwide Swiss Dam Engineering[G]. 2011.5

[2]SFOE. dam safety in Swizerland[A]. Dams in Switzerland Source for Worldwide Swiss Dam Engineering[G]. 2011.5

[3]The Federal Assembly of the Swiss Confederation. Water Retaining Facilities Act[EB/OL]. 2013.1

[4]The Swiss Federal Council. Water Retaining Facilities Ordinance[EB/OL]. 2012.10

[5]Stucky Ltd. Overview of the Swiss Dam safety Concept (Surveillance and maintenance)[R].2011.9

[6]The Swiss Committee on Dams. Methods of analysis for the prediction and the certification of dam behavior[A]. The occasion of the 21 st Congress of the International Commission on Large Dams, Montreal, June 2003.

[7]The Swiss Committee on Dams. Dam Monitoring Instrumentation Concepts, Reliablity and Redundancy[A]. The occasion of the 22nd Congress of the international Commission on Large Dams, Barcelona,June 2006.

[8]Swiss Federal Office for Civil Protection FOCP [EB/OL]. http://www.bevoelkerungsschutz.admin.ch/internet/bs/en/home/themen.html

[9]Auscultation, measurement technique and data analysis for the monitoring of dams[DB/OL].http://www.swissdams.ch/index.php/en/study-days, 2002.7

[10]Dam safety management center of M.W.R.. Survey Report of Reservoir Dam Safety Regulations revision [R]. 2014.9:21-22. 